\title{
SHORT-PHASE ANOMALIES IN INTERMITTENT BAND SWITCHING
}

\author{
T. POST, H.W. CAPEL \\ Instituut voor Theoretische Fysica, Universiteit van Amsterdam, Valckenierstraat 65, 1018 XE Amsterdam, The Netherlands
}

and

\section{J.P. VAN DER WEELE}

Centrum voor Theoretische Natuurkunde, Universiteit Twente, Postbus 217, 7500 AE Enschede, The Netherlands

Received 6 June 1988; accepted for publication 13 September 1988

Communicated by D.D. Holm

\begin{abstract}
The distribution of phase lengths $t$ for intermittent band switching is investigated for small $t$. Some typical deviations from exponential behaviour are reported, in particular the occurrence of a minimal phase length with enhanced probability.
\end{abstract}

\section{Introduction}

At present a wide variety of intermittent phenomena is known. One of the most interesting kinds of intermittency occurs in various low-dimensional maps when two chaotic bands merge [1-7].

Just after the merger of the two chaotic bands the orbit of the twice iterated map is found to spend relatively long periods in either of the former band regions, but at irregular times it switches from one region to the other. This phenomenon has been called intermittent hopping or switching and an exponential (geometrical) distribution of the periods $t$ between subsequent switches has been observed.

However, in numerical investigations we find that the phase length $t$ cannot be arbitrarily short. That is, a minimal phase length $t_{\min }$ exists, which increases when we get nearer to the merging point. Moreover, the probability distribution is strongly peaked at $t=t_{\min }$. Typical distributions of phase lengths will be shown later on in this Letter.

In this Letter we shall deal with the above shortphase phenomena, using the map

$f(x)=1-a|x|^{z}, \quad z>1$,

as an example. In section 2 we discuss the band structure of this map. In section 3 we explain the ex- istence of a minimal period $t_{\min }$ and we derive an expression for it in terms of the small parameter $\varepsilon=a-a_{1}$, where $a_{1}$ denotes the value of $a$ for which the two chaos bands merge into one. Finally, in section 4, we discuss the peak in the distribution and its dependence on $\varepsilon$ as well as on the order $z$ of the maximum.

\section{Band structure}

The map (1) exhibits a period doubling cascade as one increases the parameter $a$ from 0 to $a_{\infty}(z)$. At this value we have an orbit of period $2^{\infty}$, consisting of points which together form a Cantor set. For $a>a_{\infty}$ the attractor is composed of a collection of chaos bands, which undergoes a sequence of reverse bifurcations, or band mergings, at values ..., $a_{3}, a_{2}, a_{1}$. Here $a_{n}$ denotes the value of $a$ at which $2^{n}$ chaos bands merge into $2^{n-1}$ bands, in a pairwise manner. This is illustrated in fig. 1 for the typical case $z=2$.

For the sake of presentation we shall concentrate on the last band merging at $a=a_{1}$, although the phenomena to be described occur in exactly the same way at all the other band mergings. Just below $a=a_{1}$ the orbit of the map alternates between the two separate bands and an orbit of the twice iterated map 


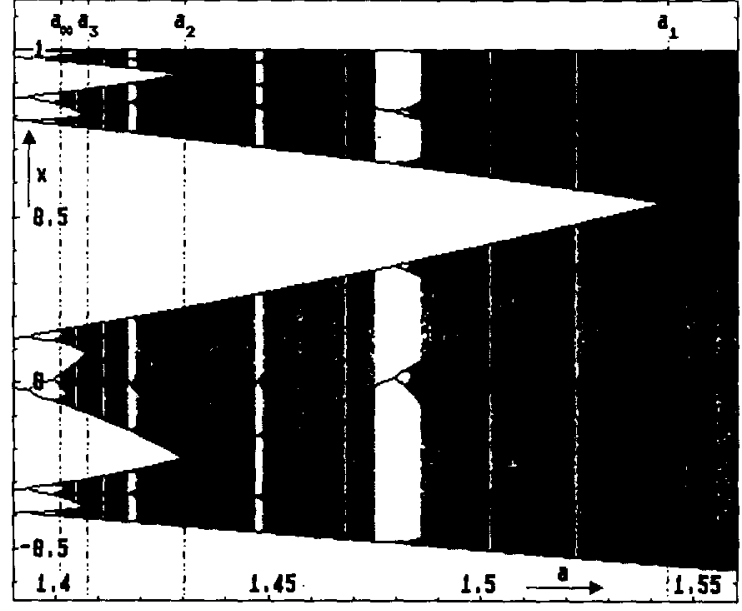

Fig. 1. The structure of the attractor for the map $f(x)=1-a x^{2}$, for $1.39<a<1.56$. We have indicated the threshold to chaos $a_{\infty}$ and the band merging values $a_{3}, a_{2}$ and $a_{1}$. (One has $a_{1}=$ 1.5436890 in this case.)

$f^{2}(x)=f(f(x))$ remains forever in either one of the bands. It is therefore convenient to consider $f^{2}(x)$ instead of the original map $f(x)$.

In figs. 2 and 3 we show $f^{2}(x)$ just before the band merging $\left(a<a_{1}\right)$ and just after it $\left(a>a_{1}\right)$. In both figures the positive fixed point $X$ of $f(x)$ is used for the construction of two square boxes. Each of these boxes contains a (sub) map similar to the original map $f(x)$ but on a smaller scale. The boxes are

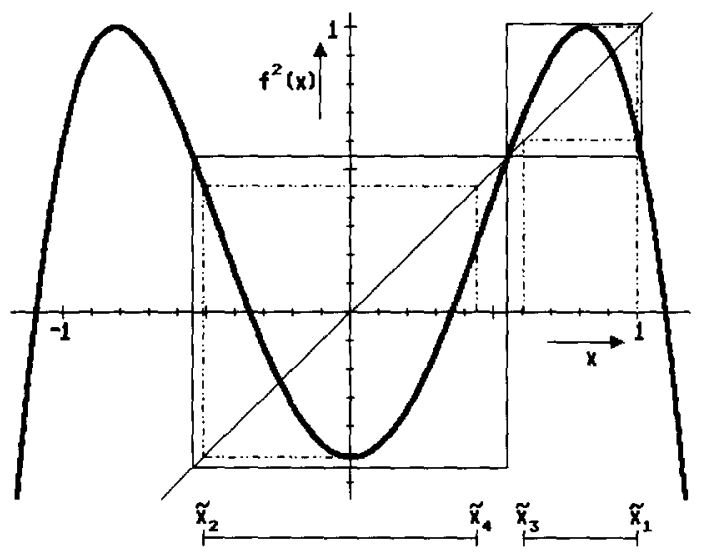

Fig. 2. The $\operatorname{map} f^{2}(x)$ for $z=2$ at $a=1.51$, before the band merging at $a=a_{1}=1.5436890$. The boundaries of the two bands are indicated by $\tilde{x}_{2}, \tilde{x}_{4}$ and $\tilde{x}_{3}, \tilde{x}_{1}$, respectively. Note that the two submaps discussed in the text are completely contained within the square boxes.

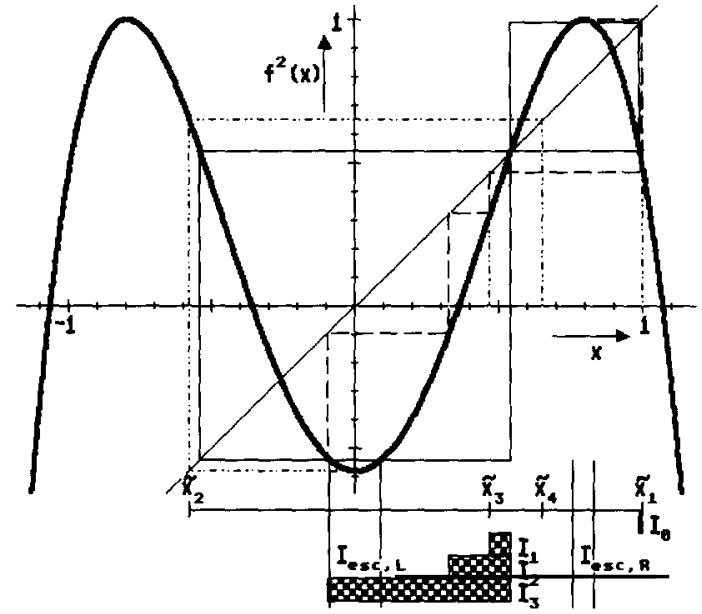

Fig. 3. The $\operatorname{map} f^{2}(x)$ for $z=2$, at $a=1.58$, after the band merging. The boundaries of the band are $\tilde{x}_{1}=1$ and $\tilde{x}_{2}=1-a$. The escape interval $I_{\text {esc, } R}$ of the right region is mapped onto the interval $I_{0}$, and then successively onto $I_{1}, I_{2}$ and $I_{3}$. Note that the interval $I_{3}$ overlaps with the escape interval $I_{\text {esc, } L}$ of the left region.

bounded by the fixed point $X$ and its pre-images un$\operatorname{der} f^{2}(x)$ at the left and the right of $X$ :

$X_{\mathrm{L}}=-X, \quad X_{\mathrm{R}}=[(1+X) / a]^{1 / z}$.

As long as the extrema of the two submaps lie inside their boxes (as in fig. 2), any orbit of $f^{2}(x)$ trapped in one of them will remain there forever. The actual chaos bands are given by the intervals $\left[\tilde{x}_{2}, \tilde{x}_{4}\right]$ and $\left[\tilde{x}_{3}, \tilde{x}_{1}\right]$, where $\tilde{x}_{n}=f^{n}(0)$ denotes the $n$th iterate of the maximum $\tilde{x}_{0}=0$, i.e. $\tilde{x}_{1}=1, \tilde{x}_{2}=1-a, \tilde{x}_{3}=$ $1-a|1-a|^{z}$, and so on. At $a=a_{1}$ the extrema of the submaps hit their boxes simultaneously, and at this moment we have $\tilde{x}_{3}=\tilde{x}_{4}=X=a_{1}-1$, which means that the band boundaries touch each other.

For $a>a_{1}$ we have the situation as shown in fig. 3 , after the band merging. The two extrema pierce through the boxes and consequently an orbit can escape from its box. For small values of $\varepsilon=a-a_{1}$ the escape regions, which are given by the intervals where $f^{2}(x)$ exceeds the boxes, are quite small, and orbits of $f^{2}(x)$ will in general remain for long times in one of the former band regions.

\section{Minimal phase length}

Let us consider the region $x>X$ in fig. 3. An orbit of $f^{2}(x)$ in the right box will switch to the left region 
$x<X$ after it gets into the escape interval $\mathrm{I}_{\text {esc, } \mathrm{R} .}$. When this happens the orbit is transferred on the next iteration into the interval $I_{0}=\left[X_{\mathrm{R}}, 1\right]$. The interval $\mathrm{I}_{0}$ is mapped by $f^{2}(x)$ to $\mathrm{I}_{1}=\left[\tilde{x}_{3}, X\right]$, then to $\mathrm{I}_{2}=\left[\tilde{x}_{5}\right.$, $X]$, then to $I_{3}=\left[\tilde{x}_{7}, X\right]$. In fig. 3 the interval $I_{3}$ overlaps with the escape interval of the left box and thus the orbit has a possibility to switch back to the right region on the second next iteration (since the escape interval is first transferred to the interval $\left[\tilde{x}_{2}, X_{\mathrm{L}}\right]$ at the left). So we observe that in this case the orbit must spend at least four iterations of $f^{2}(x)$ in the left region. We define the minimal phase length in the left region $t_{\min , \mathrm{L}}$ as the minimal number of iterations spent in that region. In general $t_{\min , \mathrm{L}}$ will depend on the value of $\varepsilon=a-a_{1}$ as well as on the order $z$ of the maximum. In the above example we have $t_{\min , \mathrm{L}}=4$.

To establish an accurate lower bound for $t_{\min }$ as a function of $\varepsilon$ we determine the minimal number $n$ of iterations of $f^{2}(x)$ needed to map $\tilde{x}_{3}$ into the escape region $I_{\text {esc, } L}$ (or to the left of it). For small values of $\varepsilon$ the region $I_{1}$ is quite narrow, with $\tilde{x}_{3}$ close to the unstable fixed point $X$, and the number of iterations can be found from

$\tilde{x}_{3+2 n}-X=\left[f^{\prime}(X)\right]^{2 n}\left(\tilde{x}_{3}-X\right)$.

The escape interval lies in a small neighbourhood of the minimum at $\tilde{x}_{0}=0$ and as soon as $\tilde{x}_{3+2 n}$ reaches this point the orbit has a possibility to escape after one extra iteration. Hence from (2) we can estimate the minimal number of iterations $n$ of $f^{2}(x)$ to get from $\tilde{x}_{3}$ to the escape region:

$n=-\frac{1}{2}=\frac{\ln \left[\left(X-\tilde{x}_{3}\right) /\left(X-\tilde{x}_{0}\right)\right]}{\ln \left|\tilde{f}^{\prime}(X)\right|}$.

Since a non-integer number of iterations has no meaning, it is understood that $n$ should be lifted to the next integer, i.e. $n \rightarrow[n+1]$.

The minimal phase length for an orbit of $f^{2}(x)$ in the left region $t_{\min , \mathrm{L}}$ is then $[n+3]$, since one iteration is needed to get into the interval $I_{1}$ and another one is needed to escape to the interval $\left[\tilde{x}_{2}, X_{\mathrm{L}}\right]$ before switching to the right region. For the right region the same arguments can be applied. However, the length of phases of $f^{2}(x)$ in the right region is always 1 iteration shorter than the length of the corresponding phases in the left region. This stems from the fact that the switching of an orbit of $f^{2}(x)$ amounts to an anomaly of the type $\mathrm{LL}$ in the alternating sequence ...LRLRLR... of an orbit of $f(x)$, where we write $L$ when an orbit point belongs to the left region and $R$ when it belongs to the right region. Anomalies of the types RR and LLL do not occur. So the minimal phase lengths in the left and the right region are related by $t_{\min , \mathrm{L}}=t_{\min , \mathrm{R}}+1$. Consequently the minimal phase length $t_{\min }$ is equal to $t_{\min , \mathrm{R}}=$ $[n+2]$.

The minimal phase length can be calculated from eq. (3), using the expressions

$$
\begin{aligned}
& X-\tilde{x}_{3}=\frac{z\left(2-a_{1}\right)}{a_{1}\left(a_{1}-1\right)} \frac{z a_{1}^{2}-2(z+1) a_{1}+2}{(z-1) a_{1}-2 z+1} \varepsilon \\
& \quad+\mathrm{O}\left(\varepsilon^{2}\right), \\
& X-\tilde{x}_{0}=a_{1}-1+\mathrm{O}(\varepsilon), \\
& f^{\prime}(X)=-\frac{z\left(2-a_{1}\right)}{a_{1}-1}+\mathrm{O}(\varepsilon) .
\end{aligned}
$$

For the case $z=2$ this yields

$t_{\min }=[2-0.965 \ln (3.43 \varepsilon)]$.

Applying eq. (7) we find the values $t_{\min }=5,7,9$, 11,14 for $\varepsilon=10^{-2}, 10^{-3}, 10^{-4}, 10^{-5}, 10^{-6}$, respectively. These values are in perfect agreement with the numerical values obtained by iteration of the interval $\mathrm{I}_{1}=\left[\tilde{x}_{3}, X\right]$.

Some of the considerations given above are reminiscent of an estimate by Grebogi et al. [8] of the phase length of the transient chaotic phase in the case of intermittent bursting. The interpretation however is quite different and the laminar phase in intermittent bursting does not show a minimal phase length effect. Further, in ref. [2] some plots of distributions of phase lengths were shown, but the minimal phase length phenomenon was not mentioned.

\section{The peak}

The peak in the distribution $P(t)$ of phase lengths $t$ at or just above $t_{\min }$ can be explained as follows. Since the escape regions for small $\varepsilon$ are quite narrow, we may assume that the points where the orbit enters the escape region $I_{\text {esc }, R}$ are uniformly distributed, that is, $\rho_{\text {esc }, \mathrm{R}}(x)$ is constant. The escape interval $I_{\text {esc, } \mathrm{R}}$ is mapped under $f^{2}(x)$ on the interval $\mathrm{I}_{0}=\left[X_{\mathbf{R}}, 1\right]$. 
The (normalized) distribution $\rho_{0}(x)$ of points mapped on this interval is obtained by applying the Frobenius-Perron operator to the (constant) distribution $\rho_{\mathrm{esc}, \mathrm{R}}(x)$, that is,

$$
\begin{aligned}
& \rho_{0}(x)=\int_{\mathrm{Iesc}, \mathrm{R}} \rho_{\mathrm{esc}, \mathrm{R}}\left(x^{\prime}\right) \delta\left(x-f^{2}\left(x^{\prime}\right)\right) \mathrm{d} x^{\prime} \\
& =z^{-1}\left(1-X_{\mathrm{R}}\right)^{-1 / z}(1-x)^{-1+1 / z}, \\
& x \in \mathrm{I}_{0} .
\end{aligned}
$$

This distribution has a cusp-type singularity at $x=1$ for $z>1$. In the limit $z \downarrow 1$ it straightens out to a uniform distribution. On the next iteration $I_{0}$ is mapped to $\mathrm{I}_{1}$, with a distribution $\rho_{1}(x)$ having a cusp-type singularity at $\tilde{x}_{3}$, i.e. at the left boundary of the interval $\mathrm{I}_{1}$. Starting from $\rho_{1}(x)$ we find after $n$ more iterations of $f^{2}(x)$ a distribution $\rho_{n+1}(x)$ with a cusptype singularity at the left boundary $\tilde{x}_{3+2 n}$ of the interval $\mathrm{I}_{n+1}$ :

$\rho_{n+1}(x)=\mathcal{N}\left(x-\tilde{x}_{3+2 n}\right)^{-1+1 / z}$,

$\mathscr{N}$ being the normalizing factor $z^{-1}\left(X-\tilde{x}_{3+2 n}\right)^{-1 / z}$.

We now consider the situation that $I_{n+1}$ is the first interval overlapping with the escape region $I_{e s c, L}$. We may anticipate that the height of the peak will oscillate as a function of $\varepsilon$, depending on the values of $\rho_{n+1}(x)$ in the escape region. More specifically, when $\varepsilon=a-a_{1}(z)$ is such that the left bound of $I_{n+1}, \tilde{x}_{3+2 n}$ falls inside the left escape region $I_{\text {esc, } L}$ we expect the probability $P_{\mathrm{L}}\left(t_{\min , \mathrm{L}}\right)$ for a phase of length $t_{\mathrm{min}, \mathrm{L}}$ in the left region to be most pronounced, yielding a maximal value for the height of the peak.

The probability $P_{L}(t)$ for a phase of length $t$ in the left region equals the probability $P_{\mathrm{R}}(t+1)$ for a phase of length $t+1$ in the right region, so we have

$P\left(t_{\min }\right)=\frac{1}{2} P_{\mathrm{L}}\left(t_{\min }+1\right)$.

We shall now calculate the maximal height of the peak at $t_{\min }$. It should be attained for values of $\varepsilon$ for which the left boundaries of $I_{n}$ and $I_{e s c, L}$ coincide. In that case the normalized distribution on $I_{n}$ can be expressed as

$\rho_{t_{\min }}(x)=z^{-1}\left(X+\frac{1}{2} L\right)^{-1 / z}\left(x+\frac{1}{2} L\right)^{-1+1 / z}$.

So the maximal probability for the minimal phase length $t_{\min }$ to occur equals

$$
\begin{aligned}
& P_{\max }\left(t_{\min }\right)=\frac{1}{2} P_{\max , \mathrm{L}}\left(t_{\min }+1\right) \\
& =\frac{1}{2} \int_{-L / 2}^{L / 2} z^{-1}\left(X+\frac{1}{2} L\right)^{-1 / z}\left(x+\frac{1}{2} L\right)^{-1+1 / z} \mathrm{~d} x \\
& =\frac{1}{2}\left(\frac{L}{X+\frac{1}{2} L}\right)^{1 / z} \sim \varepsilon^{1 / z^{2}} .
\end{aligned}
$$

In the last step we have used that $L \sim \varepsilon^{1 / z}$. On the other hand, a minimal probability $P_{\min }\left(t_{\min }\right)$ for $t_{\min }$ is expected in a situation where the left boundary of the interval $\mathrm{I}_{n}$ is just right of the escape region. From this it can be shown that $P_{\min }\left(t_{\min }\right) \sim \varepsilon^{1 / z}$, i.e. $P_{\min }\left(t_{\min }\right)$ follows the same power law as the inverse of the average phase length [1]; the maximal peak height in eq. (12), however, goes to zero with a smaller power of $\varepsilon$.

In fig. 4 we have given the numerical measurements of phase length distributions for $z=1.1, z=2$ and $z=3$, for values of $\varepsilon$ where a maximal value of $P\left(t_{\min }\right)$ occurs for $t_{\min }=6,7$ and 9 , respectively. From fig. 4 it is seen that the maximal height of the peak as compared to the inverse of the average phase length grows with increasing $z$, in agreement with the smaller power of $\varepsilon$. For actual systems such a feature may be used to gain information on the $z$ value of the underlying map. In table 1 the measured $P_{\max }\left(t_{\min }\right)$ is compared to the theoretical value according to eq. (12) and it can be concluded that the agreement is quite good.

A special situation occurs when the left boundary of $I_{n+1}$ in fig. 3 falls exactly in the middle of the left

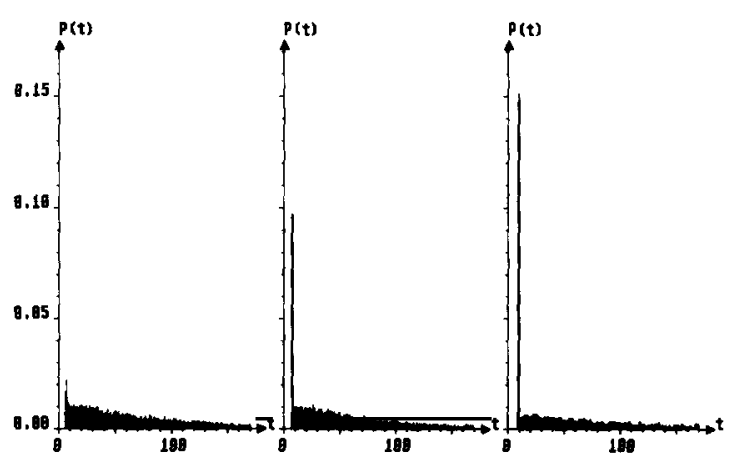

Fig. 4. Numerically obtained phase length distributions for (a) $z=1.1$ and $a=1.4377955$, (b) $z=2$ and $a=1.54424004$, (c) $z=3$ and $a=1.61804789$. 
Table 1

Comparison between prediction and measurement of $P_{\max }\left(t_{\min }\right)$ for some values of $z$ and $\varepsilon$ (see text and fig. 4).

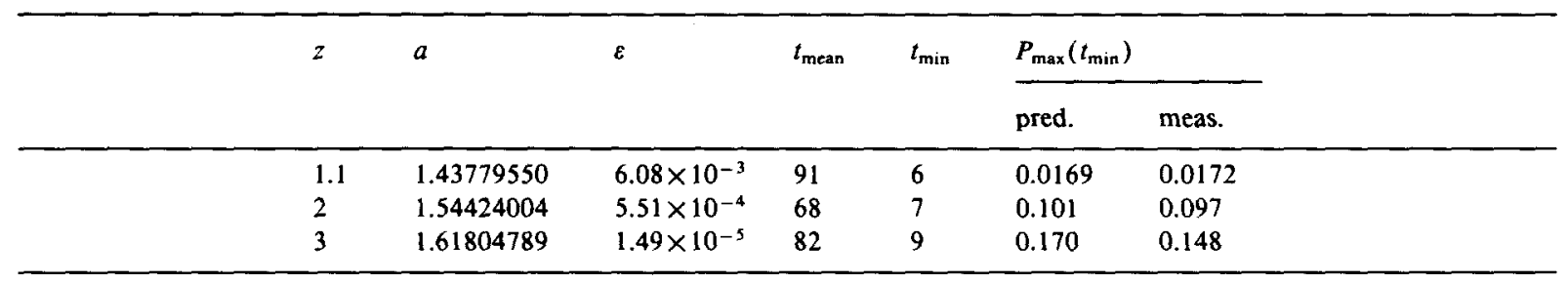

escape region. Then we have $\tilde{x}_{3+2 n}=\tilde{x}_{0}$, so at this value of $\varepsilon$ we have a superstable cycle of period $2 n+3$, and consequently $P\left(t_{\min }\right)=P\left(t_{\min }+1\right)=\frac{1}{2}$, with $t_{\min }=n+1, P(t)$ being zero for all other values of $t$. Such a cycle occurs for every value of $n$, inside a narrow window of $\varepsilon$ values. For $\varepsilon$ values corresponding to the interior part of a window one has only the two periods $t_{\min }$ and $t_{\min }+1$ occurring both with probability $\frac{1}{2}$. For $\varepsilon$ values very close to the opening and the closing of a window one may except intermittency of the Pomeau-Manneville type [9] and intermittent bursting $[8,10]$, respectively. For $\varepsilon$ values sufficiently far away from a window only the intermittent switching behaviour is left. The estimate of the minimal phase length which we have given is valid independently of the details of the windows. In the narrow subregime of intermittent bursting an analogy with earlier considerations in ref. [8] appears, because our minimal phase length in that case can be regarded as the (minimal) transient time of the bursts between the remnants of the attractor at the boundaries of the window. The interplay of the various types of intermittency will be subject of future investigation.

\section{References}

[1] S.J. Shenker and L.P. Kadanoff, J. Phys. A 14 (1981) L23.

[2] H. Fujisaka, H. Kamifukumoto and M. Inoue, Prog. Theor. Phys. Lett. 69 (1983) 333.

[3] H. Ishii, H. Fujisaka and M. Inoue, Phys. Lett. A 116 (1986) 257.

[4] O. Sporns, S. Roth and F.F. Seelig, Physica D 26 (1987) 215.

[5] R.M. Everson, Phys. Lett. A 122 (1987) 471.

[6] C. Grebogi, E. Ott, F. Romeiras and J.A. Yorke, Phys. Rev. A $36(1987) 5365$.

[7] C. Chen, G. Györgyi and G. Schmidt, Phys. Rev. A 35 (1987) 2660.

[8] C. Grebogi, E. Ott and J.A. Yorke, Physica D 7 (1983) 181.

[9] Y. Pomeau and P. Manneville, Commun. Math. Phys. 74 (1980) 189.

[10] R.W. Rollins and E.R. Hunt, Phys. Rev. A 29 (1984) 3327. 